\begin{tabular}{|c|c|}
\hline$\sqrt{\text { Version }}$ & Authors original manuscript after peer review \\
\hline Title & $\begin{array}{l}\text { Comparison of calorimetric and dielectric single component glass } \\
\text { transitions in PtBS-PI blends }\end{array}$ \\
\hline Authors & Arrese-Igor, Silvia; Alegria, Angel; J.; Colmenero, Juan \\
\hline & Publisher data \\
\hline Issue date & 2010 \\
\hline Publisher & American Chemical Society \\
\hline Gitation & Macromolecules 43(15): 6406-6413 (2010) \\
\hline Publisher Version & http://dx.doi.org/10.1021/ma101022a \\
\hline URI: & http://hdl.handle.net/10261/44200 \\
\hline DOI: & http://dx.doi.org/10.1021/ma101022a \\
\hline ISSN & 0024-9297 \\
\hline E-ISSN: & $1520-5835$ \\
\hline
\end{tabular}




\title{
Comparison of calorimetric and dielectric single component glass transitions in PtBS-PI blends
}

\author{
S. Arrese-Igor ${ }^{1}$, A. Alegría ${ }^{1,2}$, J. Colmenero ${ }^{1,2,3}$ \\ 1 Centro de Física de Materiales (CSIC-UPV/EHU), \\ Apartado 1072, 20080 San Sebastián, Spain \\ 2 Departamento de Física de Materiales UPV/EHU, \\ Apartado 1072, 20080 San Sebastián, Spain and \\ 3 Materials Physics Center (MPC), and Donostia International Physics Center, \\ Paseo Manuel de Lardizabal 4, 20018 San Sebastián, Spain
}

(Dated: June 30, 2010)

Miscible blends of polyisoprene (PI) and polytertbutylstyrene (PtBS) have been studied over the entire composition range by means of differential scanning calorimetry (DSC) and dielectric spectroscopy (DS) techniques. Blends with two different molecular weight PtBS, $M_{n}=1300$ and $M_{n}=2300$, and a $M_{n}=2700$ PI have been investigated. The molecular weight of all the polymers were chosen in order to be well bellow the entanglement limit for PI. Calorimetric measurements confirm the presence of two component glass transitions $\left(T_{g}\right)$ as previously reported. The comparison of the component's $T_{g}$ observed by DSC and the segmental dynamics directly measured for PI by DS, evidence the equivalence between the calorimetric effective $T_{g}$ and the freezing of the segmental relaxation of PI around $10^{2} s$ observed by DS. Fitting of the experimental $T_{g}$ values to the Lodge-McLeish model gives self concentration values that depend on the molecular weight of the PtBS. Finally, 
blends with the lowest PI content exhibit unusual behavior which is discussed in the framework of non-equilibrium effects. 


\section{INTRODUCTION}

Dynamics in polymer blends is a very important topic, not only from a fundamental point of view, but also for technological applications. The mixing of two compatible polymers is one of the most cost effective ways to tune the properties of available polymeric materials to meet application needs. A limited knowledge of the blending phenomenon restricts the advance in the pursuit of taylor-made materials so that a deep molecular level understanding is crucial. Miscible polymer blends have been known for some time to exhibit striking dynamic phenomena (see e.g. review ${ }^{1}$ and references herein). Concerning the segmental dynamics driving the so-called $\alpha$-relaxation, two main effects are well established by extensive experimental studies: i) the isolated dynamical response of each component broadens with respect to the pure polymer behavior, this effect being strongly dependent on temperature (tends to disappear at high T) and more evident for the component with the lower glass transition temperature, $T_{g}$; and ii) two distinct mean relaxation times are usually found in the blend, each of them corresponding to the dynamics of each component modified by blending, what is usually called 'dynamic heterogeneity' (see some representative references ${ }^{2-7}$ ).

It is generally accepted that the unusual properties of miscible polymer blends result to the distinct segmental dynamics of its components. Thermally driven concentration fluctuations and self-concentration effects are thought to be the origin of the mentioned dynamic heterogeneity. For a given component A, it is believed that its particular dynamics is controlled by the local composition of the component, $\phi_{i}^{A}$, in a volume $\mathrm{V}$ around the segment $i$ of $\mathrm{A}$, which in general, will be different from its average (macroscopic) composition $<\phi^{A}>$. As a consequence of the different local compositions seen by each component in the blend $\phi_{i}^{A} \neq\left(1-\phi_{j}^{B}\right)$, they show distinct effective glass transition, $T_{g}^{e f f}$, whose value will 
be closer to its homopolymer value than the value predicted by a simple mixing rule for the blend's macroscopic composition.

On the other hand, the observation of a single glass-transition temperature by calorimetry has been, perhaps, the most extended criterion of miscibility used in the literature for many years, a concept which openly oppose to the dynamic heterogeneity ideas presented above. Although there are several pioneering works strongly supporting the presence of distinct effective glass transitions for each component in miscible polymer mixtures by calorimetry ${ }^{8-13}$, the question of resolving dynamic heterogeneity effects by this technique is not still out of debate. Very recently J. Zhao et al ${ }^{12}$ quite clearly show this phenomenology for Polyisoprene $\left(\overline{M_{n}}=3000\right.$ to 17000$)$ and Poly-tertbutylstyrene $\left(\overline{M_{n}}=32000\right)$ mixtures characterized by an extraordinarily large $T_{g}$ difference between the two homopolymers.

Dielectric Spectroscopy (DS) is a well established tool to study polymer dynamics. Moreover, when studying polymer mixtures if only one of the two components is dielectrically active it becomes selective, and allows to measure single component dynamics in a broad frequency range. In this work we compare the equilibrium-non equilibrium glass transitions observed by calorimetric measurements for each component with the PI's segmental dynamics accessible by dielectric techniques for Polyisoprene-Polytertbutylstyrene blends. The molecular weight of the polymers used were $\overline{M_{n}}=2700$ for Polyisoprene and $\overline{M_{n}}=1300$ and $\overline{M_{n}}=2300$ for Poly-tertbutylstyrene. With this choice the difference between the $T_{g} \mathrm{~s}$ of the two homopolymers is still high enough to expect distinguishable effective glass transitions by calorimetric measurements, whereas the molecular weights of both components are well below the entanglement limit for PI, which is around $\overline{M_{n}}=4000$. This is of great importance to study the effect of blending on the chain dynamics (the so called Normal Mode) regardless any entanglement effect, which will be the subject of a separate communication. 


\section{EXPERIMENTAL}

\section{A. Samples}

Samples were prepared starting from commercially available pure homopolymers Polyisoprene and Poly-tert-butylstyrene (purchased from Polymer Source). The low $-T_{g}$ component of the blends was Polyisoprene $(80 \%$ cis 1,$4 ; 15 \%$ trans 1,$4 ; 5 \%$ trans 3,4$)$ with $\overline{M_{n}}=2700$ and $\overline{M w} / \overline{M n}=1.07$ (PI2700 from now on) and a glass transition temperature $T_{g}=204 \mathrm{~K}$ determined by differential scanning calorimetry. Two Poly-tert-butylstyrenes with methyl groups at the chain ends and different molecular weights were used as high- $T_{g}$ components: PtBS1300 $(\overline{M n}=1300 ; \overline{M w} / \overline{M n}=1.08)$ and PtBS2300 $(\overline{M n}=2300 ; \overline{M w} / \overline{M n}=1.06)$ with $T_{g}=330 \mathrm{~K}$ and $T_{g}=373 \mathrm{~K}$ respectively. Low Molecular weights for all the polymers were chosen in order to be well below the entanglement limit for PI. Starting from the pure homopolymers, blends with several PI concentrations in weight content, namely, $80 \%, 50 \%$, $35 \%$, and $20 \%$ for blends containing PtBS1300, and 80\%, 65\%, 43\%, 35\%, and $20 \%$ for blends containing PtBS2300, were prepared by solution casting from Toluene solutions. Prior to measurements samples were vacuum dried at temperatures above glass transition to completely eliminate any trace of solvent. The so obtained blends were transparent and show no crystallization or melting by calorimetry. The miscibility of PI-PtBS blends has been studied by small-angle neutron scattering and small-angle X-Ray scattering by Yurekli et al. ${ }^{14}$ and Watanabe et al. ${ }^{15}$ respectively. The system shows a LCST behavior but remains miscible in the whole temperature range examined by Yurekli - up to 443K - and Watanabe - up to 343K-. The miscibility of the blends is further supported by the calorimetric and dielectric measurements herein.

Finally, following the mentioned procedure, additional blends with a high molecular 
weight PI, $T_{g}=211, \overline{M n}=76500$ and $\overline{M w} / \overline{M n}=1.07$ (PI76500 from now on) and high PtBS concentration (80\%) were prepared to study the dielectric $\alpha$-relaxation of both PtBS and PI components in the blend.

\section{B. Differential Scanning calorimetry}

A differential scanning calorimeter TA Instrument Q2000 was used both in conventional and "heat only" modulation mode to measure the calorimetric glass transition temperature of the blends. Samples of about $10 \mathrm{mg}$ were sealed in aluminum pans and heated at a $20 \mathrm{~K} / \mathrm{min}$ rate for conventional, and at an average $2 \mathrm{~K} / \mathrm{min}$ rate for modulated experiments with a temperature modulation amplitude and period of $\pm 0.32 \mathrm{~K}$ and 60 s respectively. Higher heating rate in conventional scans provides better sensitivity whereas modulated method allows to separate changes in the heat flow due to variations in the heat capacity of the sample from other type of heat contributions, that is, the so called reversing and nonreversing flows. Both, conventionally measured heat flow and reversing heat flow measured by modulated experiments provided undistinguishable results for all the samples within the accuracy of the method.

The calorimetric glass transitions of the studied blends are asymmetric and very broad, extending over a T-range larger than $60 \mathrm{~K}$ for the blends with PI content lower than $50 \%$. As a consequence, the determination of a glass transition temperature from the heat flow curves by the usual tangent method is subjected to large uncertainties. Alternatively, the temperature derivative of the calorimetric signal provides a much more sensitive and easier way to analyze changes in the heat flow. In particular, when the calorimetric spectrum is asymmetric the tangent method tends to give glass transition values close to the maxima of the derivative, underestimating the change of specific heat occurring at the asymmetric 

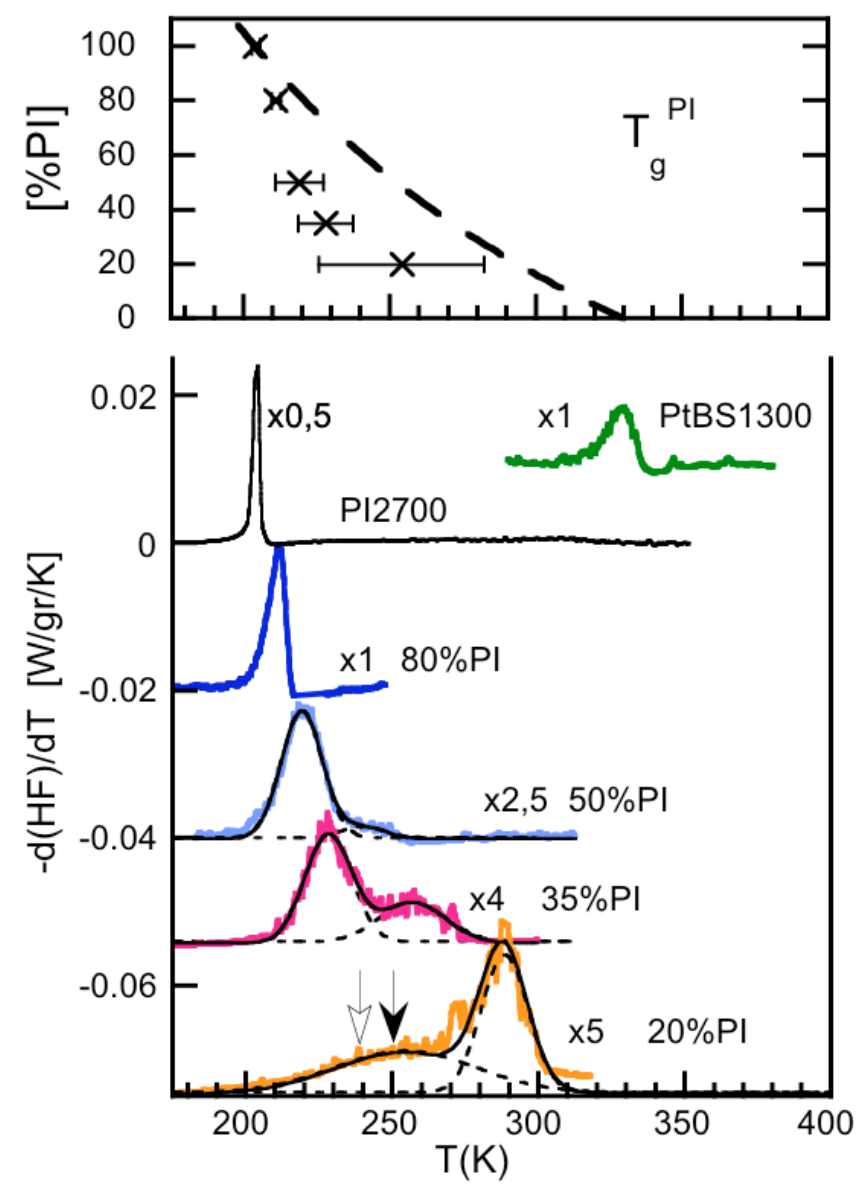

FIG. 1: Lower panel: Derivative of the Heat Flow with respect to T for PI2700 and PtBS1300 homopolimers and their blends at different compositions (color on line). The intensity of the curves was multiplied by $0.5,2.5,4$, and 5 , for $100,50,35$, and $20 \%$ samples respectively. In addition, curves have been shifted in the $y$ axis for clearness. Continuous black lines represent fits to two gaussian functions and dashed lines individual components of the fitting. Upper panel: Glass transition temperature of PI component in the blend as a function of PI content (crosses); horizontal bars represent full width at half maximum of the gaussian fits; dashed line represents the expected global glass transition temperature for the blend according to a simple mixing rule. 

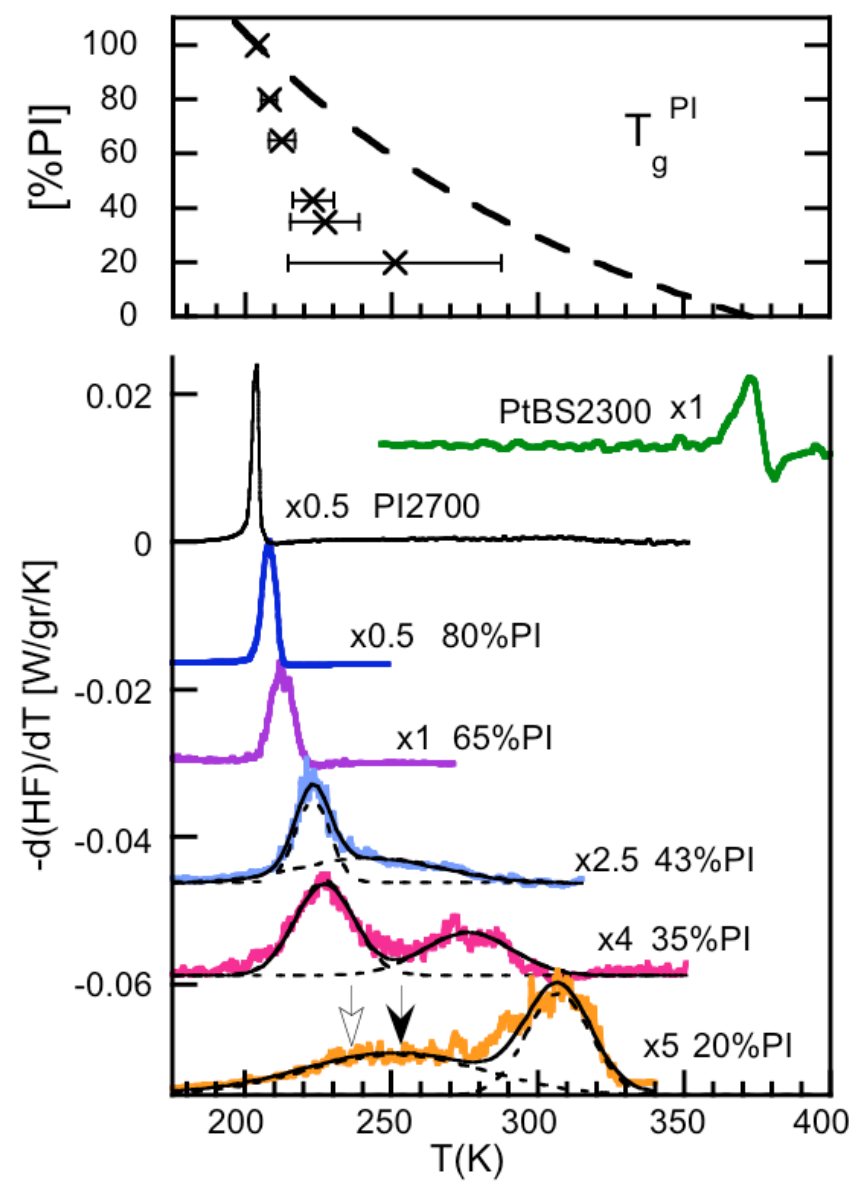

FIG. 2: Lower panel: Derivative of the Heat Flow with respect to T for PI2700 and PtBS2300 homopolimers and their blends at different compositions (color on line). The intensity of the curves was multiplied by $0.5,0.5,2.5,4$, and 5 , for $100,80,43,35$, and $20 \%$ samples respectively. In addition, curves have been shifted in the y axis for clearness. Continuous black lines represent fits to two gaussian functions and dashed lines individual components of the fitting. Upper panel: Glass transition temperature of PI component in the blend as a function of PI content (crosses); horizontal bars represent full width at half maximum of the gaussian fits; dashed line represents the expected global glass transition temperature for the blend according to a simple mixing rule. 
end of the spectrum. Figures 1 and 2 show the derivative of the heat flow recorded at $20 \mathrm{~K} / \mathrm{min}$ for different blend compositions, where the mentioned extension of the calorimetric transition is evident. Moreover, two distinct glass transition ranges can be envisaged from the progressive high- $\mathrm{T}$ asymmetric broadening of the signal as PI content decreases, which eventually results in the development of two well resolved peaks. The smooth variation of the different component peak position between those for the homopolymers corroborates the miscibility of the system.

In order to determine each component glass transition from calorimetric data we have fitted the measured derivative of the heat flow (Figures 1 and 2) with two overlaping gaussian functions. For PI concentrations above $50 \%$ where two peaks are not well resolved, the glass transition for PI has been determined from the gaussian fit of the low-T side of the peak only. The so obtained glass transition temperatures for the different components and blend compositions are included in Table 1 and in the upper panel of Figures 1 and 2.

\section{Dielectric Spectroscopy}

A broadband dielectric spectrometer, Novocontrol alpha-A analyser, was used to isothermaly measure the complex dielectric function $\epsilon^{*}(\omega)=\epsilon^{\prime}(\omega)-i \epsilon^{\prime \prime}(\omega)$ between 130 and 350K, and in the $10^{-2}$ to $10^{7} \mathrm{~Hz}$ frequency range, $\omega=2 \pi f$, by placing samples between parallel gold-plated electrodes of $30 \mathrm{~mm}$ diameter. The temperature stability was better than $\pm 0.2 \mathrm{~K}$. Dielectric losses of PI are almost two orders of magnitude larger than that of PtBS. As a result, when dielectricaly measuring PI-PtBS blends, we will be principally sensible to the PI component dynamics in the blend.

The measured spectra in general show two partially overlapped relaxation processes corresponding to the main chain (normal mode) and the segmental $(\alpha)$ relaxation of PI2700 in 

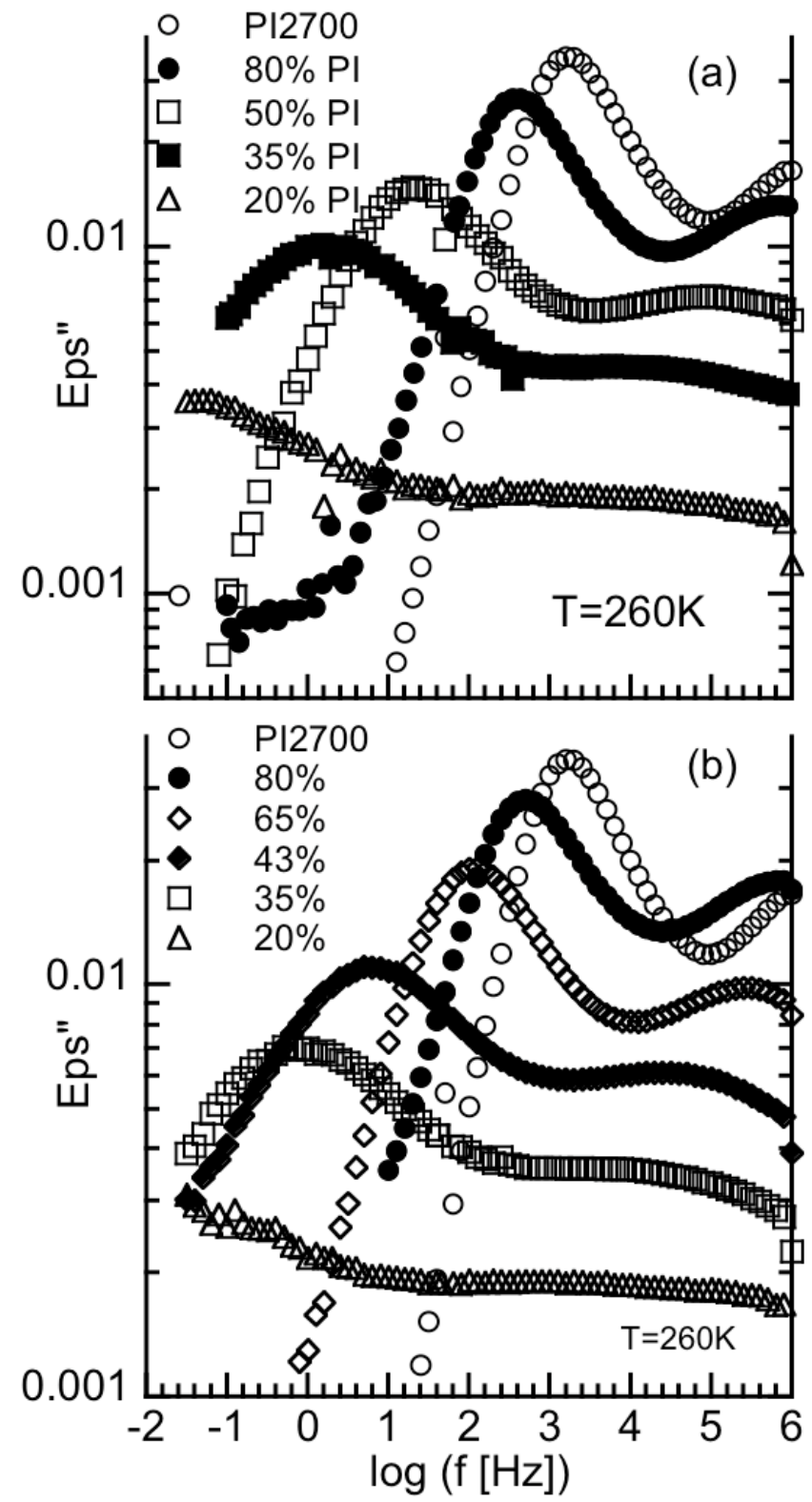

FIG. 3: Imaginary part of the dielectric permitivity at 260K for PI2700-PtBS1300 and PI2700PtBS2300 blends -panels (a) and (b) respectively- at different compositions.

the blends (see Figure 3). With decreasing PI content, the dielectric loss intensity of these 
relaxations decreases and its characteristic frequencies become lower and lower, as expected for miscible blends. Once again the smooth variation of the signals confirms the miscibility of the system, as phase separation for the intermediate compositions $50-43 \%$ would manifest in characteristic times for PI motions similar to richer PI compositions like 80 or $65 \%$. Regarding the shape of the relaxations, we found a gradual broadening of both processes with decreasing PI content, which makes the $\alpha$-relaxation of lower PI content blends to be less and less pronounced. In the following we will focus on the study of the $\alpha$-relaxation process. The complete analysis and characterization of the normal mode relaxation will be the subject of a separated communication.

The position of the maxima of $\epsilon$ " $(\omega)$ as a function of frequency at a constant temperature gives a direct measure of the characteristic time of the motion involved. Characteristic frequencies for the $\alpha$-relaxation of PI component in the blend were determined from the position of the maxima of the relaxation loss $\left(2 \pi f_{\max }=1 / \tau\right)$ by a fitting procedure which involved two Havriliak-Negami (HN) functions.

$$
\epsilon^{*}(\omega)=H N_{\text {chain }}+H N_{\alpha}
$$

where,

$$
H N=\frac{\Delta \epsilon}{\left(1+\left(i \omega \tau_{H N}\right)^{\beta}\right)^{\gamma}}
$$

and $\tau_{H N}$ stands for Havriliak-Negami's characteristic time, related to maximum frequency by,

$$
\left(2 \pi f_{\max }\right)^{-1}=\tau_{H N}\left[\frac{\sin \left[\frac{\beta \gamma \pi}{2(\gamma+1)}\right]}{\sin \left[\frac{\beta \pi}{2(\gamma+1)}\right]}\right]^{1 / \beta} .
$$

The obtained $\log f_{\max }$ values for PI's $\alpha$-relaxation are represented by filled symbols in Figure 4 for the different blends.

The segmental relaxation of the PtBS component in the blend produces a very low in- 

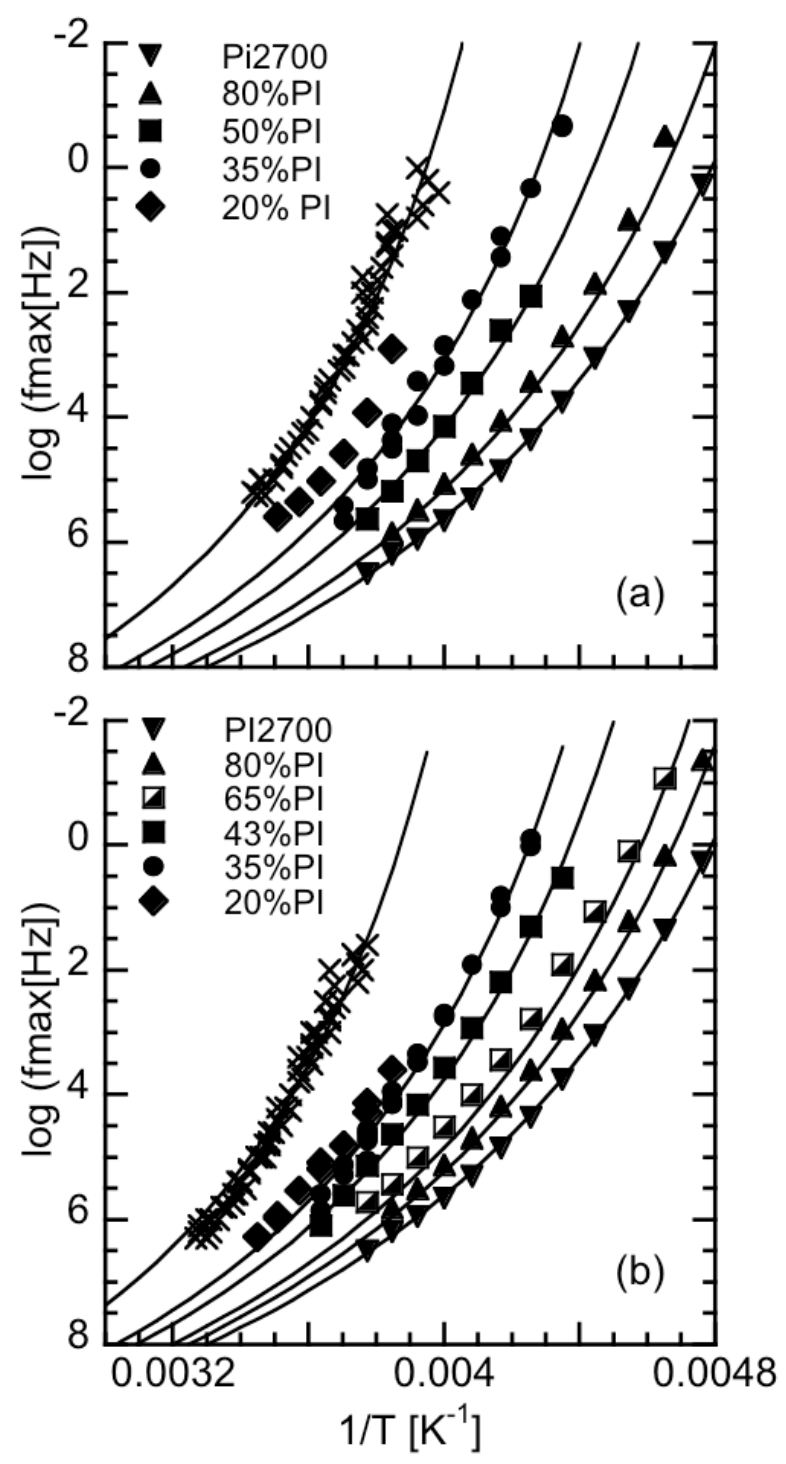

FIG. 4: Experimental $\log f_{\max }$ of the PI dielectric $\alpha$-relaxation for pure PI2700 and its blends with PtBS1300 and PtBS2300 -panels (a) and (b) respectively- at different weight percentages. Solid lines represent the prediction from the $T_{g, D S C}^{P I}$ obtained by DSC. Crosses represent the characteristic times obtained from isochronal representation of the data for $20 \%$ PI blends.

tensity dielectric loss, orders of magnitude lower than that of PI, and in a frequency range close to PI2700's normal mode. In order to have access to this low signal and study PtBS's 
$\alpha$-relaxation by dielectric spectroscopy, blends with high PtBS content and a high molecular weight PI were prepared. Thanks to the longer chain length of PI76500, the normal mode relaxation of PI moves to much lower frequencies bringing to light for this sample with high PtBS content the small contribution from the $\alpha$-relaxation of the PtBS component in the blend, otherwise masked by the PI2700's normal mode (see upper panel of Figure 5). In The lower panel of Figure 5 filled symbols represent the characteristic frequency of the PtBS $\alpha$-relaxation for pure PtBS1300 (squares) and in its blend with 20\%PI76500 (circles).

\section{DISCUSSION}

\section{A. Individual component glass transitions}

1. Individual component glass transitions by DSC

The calorimetric glass transition of the studied blends are asymmetric and very broad, extending over a T-range larger than $60 \mathrm{~K}$ for the blends with PI content lower than $50 \%$. This phenomenology has often been reported for polymer blends in general, and in particular, for blends with high dynamical heterogeneity as those studied here. Moreover, there is a progressive asymmetric broadening of the high-T signal as PI content decreases which eventually results in the development of two well resolved peaks. The existence of two different dynamics in blends, each corresponding to the segmental dynamics of individual components, is a well established result. Therefore, it is natural to identify the double peak structure of the calorimetric spectra with the effective glass transitions of each component in the blend. The smooth variation of the individual component peak intensity and position between those for the pure homopolymers supports the previous statement. As it can be seen in Figures 1 and 2, the individual calorimetric glass transitions of the different components in 

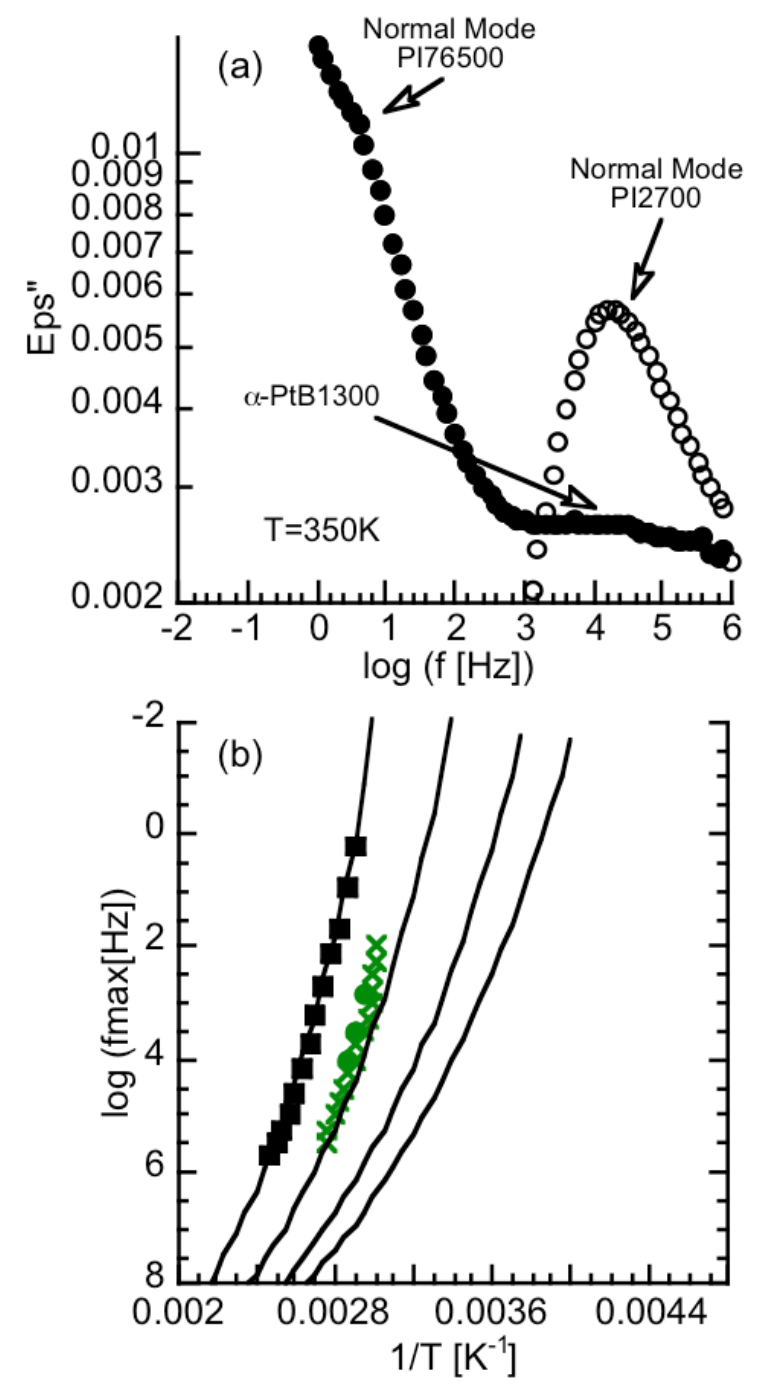

FIG. 5: Upper panel: Imaginary part of the dielectric permitivity of 20\%PI76500-PtBS1300 (filled symbols) and 20\%PI2700-PtBS1300 (empty symbols) blends at 350K. Lower panel: Experimental $\log f_{\max }$ of the PtBS segmental dielectric relaxation for pure PtBS1300 (squares) and its blend with 20\% PI76500 (circles: maxima from isothermal representation, crosses: position of the maxima from an isochronal representation). Solid lines represent the prediction from the $T_{g, D S C}^{P t B S}$ obtained by DSC. 
the blend are not only shifted but also broadened relative to the homopolymer calorimetric signal, as a result, when the relative dynamic heterogeneity of the polymers is not very high a single broad glass transition is likely to be observed. Finally, as we will present in the next section, the comparison of the so obtained calorimetric effective glass transition and the segmental dynamics measured by DS for PI evidences that the double peak structure is not an artifact, but certainly reflects the dynamic heterogeneity of the blends.

\section{Comparison of DSC and DS results}

Due to the much higher dielectric relaxation strength of PI, dielectric spectroscopy provides a selective way to characterize PI segmental dynamics in these blends. Therefore, it is of great interest to address the question of how do the peak temperatures deduced from DSC analysis (which we have identified with the individual component glass transitions in the blend) compare with the segmental relaxation dynamics of PI in the blend directly measured by dielectric techniques. In order to make such a comparison we have calculated Vogel-Fulcher-Tamman (VFT) curves

$$
\log f_{\text {max }}=\log f_{\infty}-\frac{B}{\left(T-T_{o}\right)}
$$

for the $\alpha$-relaxation of PI in the blends starting from the component glass transition temperatures deduced from DSC $\left(T_{g, D S C}^{c o m p}\right)$ assuming:

$$
T_{g}^{h o m o}-T_{g}^{c o m p}=T_{o}^{h o m o}-T_{o}^{c o m p}
$$

where $T_{o}$ is the Volgel-Fulcher-Tamman (VFT) temperature, homo and comp stand for pure homopolymer and blend component respectively. In addition, $\log f_{\infty}$ and $B$ parameters were fixed to those obtained for the pure homopolymer. In general, the so obtained predictions for 
the PI segmental dynamics show excellent agreement with the dielectric experimental data (see Figure 4). We stress that solid lines in Figure 4 are not VFT fittings to dielectric data but the prediction from $T_{g, D S C}^{P I}$ according to the procedure described above. The PI's component glass transition temperature observed by DSC for the most diluted PI composition is higher than that expected by following the microscopic $\alpha$-relaxation dynamics of PI component by DS. We will separately address this question in the framework of non-equilibrium effects through section III C. Analogous calculation and comparison (when data is available) for the PtBS $\alpha$-relaxation and $T_{g}^{P t B S}$ by DSC also shows good agreement as shown in the lower panel of Figure 5.

Following a common practice in the analysis of pure homopolymers, we can play the other way round and predict PI component glass transition temperatures in the blend from dielectric data $\left(T_{g, D S}^{P I}\right)$. The characteristic frequencies for the $\alpha$-relaxation of PI component in the blend have been fitted to a VFT law and the component glass transition temperature estimated from the extrapolation of the fitting at $\log f\left(T_{g}\right)=-2$. The so obtained $T_{g, D S}^{P I}$ temperatures are depicted in Figure 6 (crosses) together with $T_{g, D S C}^{P I}$ ones (circles) for different blends as a function of PI2700 content. As we can see, $T_{g, D S}^{P I}$ values obtained from dielectric measurements predict reasonably well the calorimetric behavior. The goodness of the comparison makes it clear that the dynamic heterogeneity for the different components of a blend, that is, the concept of different mobility and effective glass transition for each component in the blend, also has its counterpart in calorimetric macroscopic measurements as long as the difference in $T_{g} \mathrm{~s}$ is large enough to be detected within the resolution limits of the technique. These findings corroborate the unsuitability of both miscibility and inmiscibility criteria based on the existence of a single or double calorimetric transitions respectively ${ }^{11,12}$. 

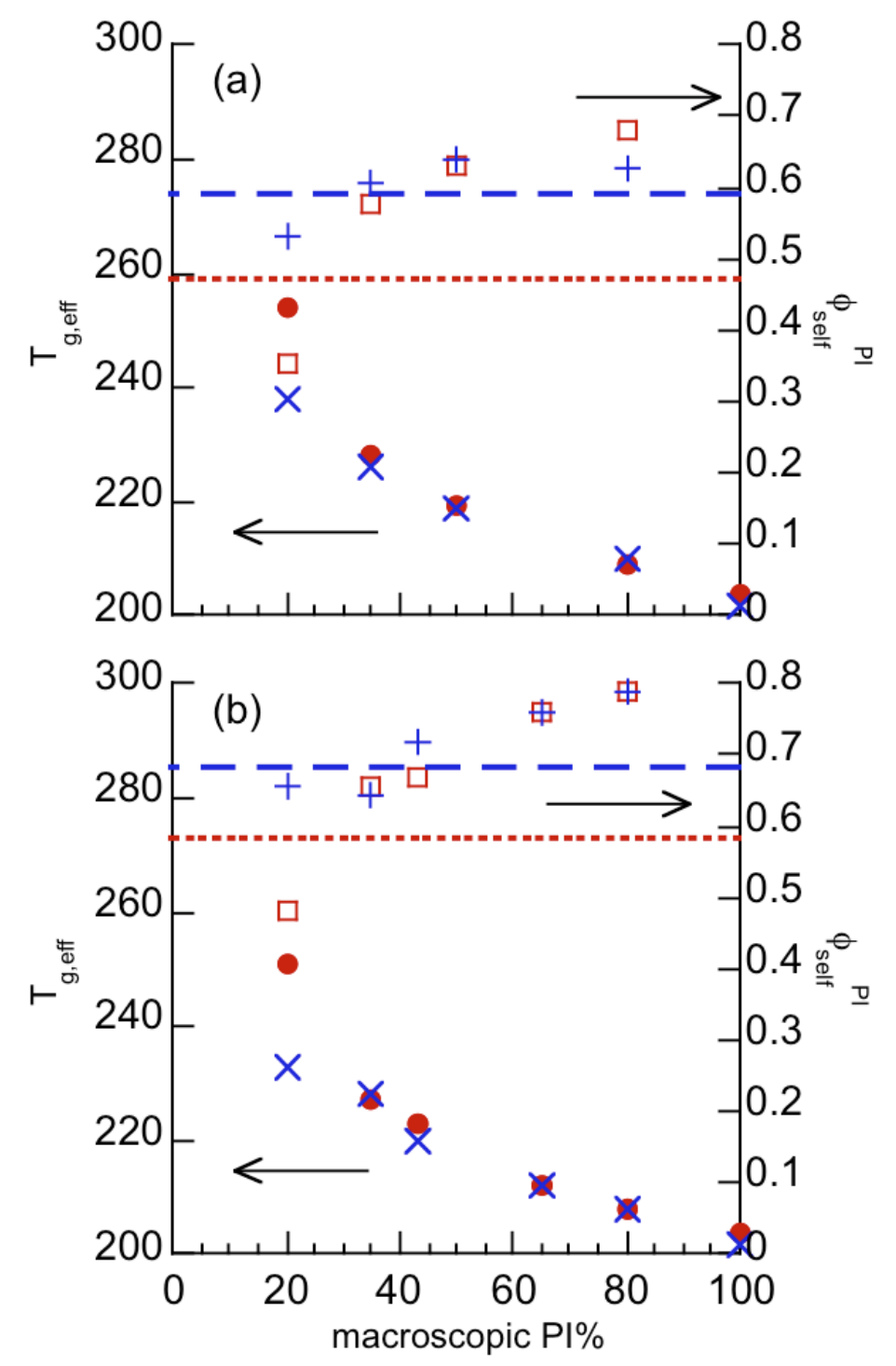

FIG. 6: Single component glass transitions $T_{g, D S C}^{P I}, T_{g, D S}^{P I}$ and self concentration $\phi_{\text {self }}$ of PI2700 in its blends with PtBS1300 and PtBS2300 -panels (a) and (b) respectively- obtained from both DSC (red: circles $T_{g}$, squares $\phi_{\text {self }}$ ) and DS (blue: crosses $T_{g}$, plusses $\phi_{\text {self }}$ ) measurements. 


\section{B. Self concentration}

As commented in the introduction, there are two main candidates to explain the variation in local composition of polymer blends: thermally driven concentration fluctuations and selfconcentration effects. The idea underlying self-concentration is that the local concentration around one segment of any component will be always richer in that component due to chain connectivity. Among those models devoted to self concentration effects, the so-called Lodge-McLeish model (LML) posses the most elaborated formulation which opens the way for some calculation and experimental test. The LML model defines the local (effective) concentration in a volume $\mathrm{V}$ around a given segment of component $\mathrm{A}$, as:

$$
\phi_{\text {eff }}^{A}=\phi_{\text {self }}^{A}+\left(1-\phi_{\text {self }}^{A}\right) \chi_{A}
$$

where $\chi_{A}$ is the macroscopic concentration of component $\mathrm{A}$, and $\phi_{\text {self }}$ is a polymer specific parameter linked to the relevant length scale of its segmental dynamics called "self - concentration". In particular, the model assumes that the glass transition of component A in the blend will be that corresponding to the "average blend $T_{g}$ " evaluated at the effective or local concentration $\phi_{\text {self }, A}$.

$$
T_{g}^{\text {compA }}(\chi)=\left.T_{g}(\chi)\right|_{\chi=\phi_{\text {self }}^{A}}
$$

It is worth mentioning that in the framework of the existence of two $T_{g} \mathrm{~s}$ in a miscible blend, the $T_{g}^{c o m p A}$ in equation 7 should be considered as some kind of ill-defined average $T_{g}$ of the blend. It is in some way ironic that a model proposing two $T_{g} \mathrm{~s}$ in a miscible blend operatively uses an expression as equation 7 , which is based on the existence of a $T_{g}(\chi)$, i.e., on the old idea that miscibility means only one $T_{g}(\chi)$. In any case, we have followed the standard procedure reported in the literature to calculate the self-concentration of the LML model. 
By using the formulation above, together with a simple mixing rule to calculate the glass transition temperature,

$$
\frac{1}{T_{g}^{\text {comp }, A}}=\frac{\phi_{e f f}^{A}}{T_{g}^{\text {homoA }}}+\frac{1-\phi_{e f f}^{A}}{T_{g}^{\text {homoB }}}
$$

and the experimental values obtained for component glass transitions by DS and DSC, we have calculated the self concentration of PI2700, $\phi_{\text {self }}^{P I}$ : (i) for each technique (DS and DSC) and each blend composition separately; and (ii) by fitting $T_{g}^{P I}$ as a function of PI content to a combination of equations 6 and 8 for each technique (DS and DSC). The global fitting of $T_{g}^{P I}$ temperatures [method (ii)] leads to $\phi_{\text {self }}^{P I}$ values of 0.47 and 0.57 in PtBS1300 blends and 0.58 and 0.67 in PtBS2300 blends for DSC and DS data collections respectively and have been indicated by dotted -DSC- and dashed -DS- lines in Figure 6. The point by point calculated $\phi_{\text {self }}^{P I}$ values (squares DSC and plusses DS in Figure 6) more or less scatter around the globally fitted value for DS results, while they stay slightly far from the fitted value for DSC. This is probably due to the anomalously high $T_{g, D S C}^{P I}$ observed by DSC in comparison with that inferred from the segmental relaxation dynamics of PI by DS in $20 \%$ PI blends. We will come back to this question later in section III C.

LML model estimates a constant self concentration value for PI, $\phi_{\text {self }}^{P I}=0.45$, based on Kuhn's volume ${ }^{16}$. Experimentally, very different self concentration values have been reported for PI when blended with different partners: $\phi_{\text {self }}^{P I}=0.33^{17}$ and $\phi_{\text {self }}^{P I}=0.20^{18}$ in PI/PS blends; $\phi_{\text {self }}^{P I}=0.41^{18}$ in PI/PVE; and $\phi_{\text {self }}^{P I}=0.85^{18}$ in PI/PB, among others. A partner dependent $\phi_{\text {self }}^{P E O}$ has also been observed for a series of PEO blends with PVAc, PMMA, or $\mathrm{PLA}^{11}$. At the light of the results obtained here it seems that self concentration may not only change with blending partner, but also with the molecular weight of the blending partner. Point by point calculated self concentration averages were $<\phi_{\text {self,DSC }}^{P I}>=0.56 \pm 0.14($ where \pm 0.14 stands for standard deviation) and $\left\langle\phi_{\text {self,DS }}^{P I}>=0.60 \pm 0.05\right.$ when blended 
with PtBS1300, while much a higher values $\left\langle\phi_{\text {self }, D S C}^{P I}\right\rangle=0.67 \pm 0.12$ and $\left.<\phi_{\text {self,DS }}^{P I}\right\rangle=$ $0.71 \pm 0.06$ are obtained for blends with PtBS2300. Ediger et al. reported a self concentration value of $\phi_{\text {self }}^{P I}=0.63^{12}$ as well for PI blended with a $M_{n}=32000$ PtBS.

The $\phi_{\text {self }}^{P I}$ value determined by fitting the data for PI-PtBS is significantly larger than that predicted by LML model or those experimentally determined for PI in other blends. It has been argued that large $\phi_{\text {self }}$ values were reported for blends with large homopolymer $T_{g}$ differences, e.g. $\Delta T_{g}=197 \mathrm{~K}$ and $\phi_{\text {self }}^{P E O}=0.55$ in PEO $/ \mathrm{PMMA}$ blends ${ }^{11}$, or $\Delta T_{g}=96 \mathrm{~K}$ and $\phi_{\text {self }}^{P E O}=0.64$ in $\mathrm{PEO} / \mathrm{PVAc}^{11}$. However, other cases could be mentioned where similar $\Delta T_{g}$ do not produce such a large $\phi_{\text {self }}\left(\Delta T_{g}=111 \mathrm{~K}\right.$ and $\phi_{\text {self }}^{P E O}=0.37$ in PEO/PLA blends $\left.{ }^{11}\right)$, or where high $\phi_{\text {self }}$ values arise in blends with small dynamic heterogeneity $\left(\Delta T_{g}=20 \mathrm{~K}\right.$ and $\phi_{\text {self }}^{P E O}=0.85$ in PI/PB blends $\left.{ }^{18}\right)$. The idea that a large $\Delta T_{g}$ leads to a large $\phi_{\text {self }}$ for the low- $T_{g}$ (fast) component as a result of the non-equilibrium state produced when the high$T_{g}$ (slow) component freezes, is neither consistent with the fact that $\phi_{\text {self }}$ does not clearly depend on the low- $T_{g}$ component concentration. In the blends studied here in particular, the trend, if any, would be a decrease of $\phi_{\text {self }}^{P I}$ for blends with the lowest PI content. This is against the expected trend for non-equilibrium effects, which should be more prominent the more dilute and the higher the dynamic heterogeneity between components. Furthermore, for the blends with PI content $\gtrsim 50 \%$ studied in this work (which also exhibit large $\phi_{\text {self }}^{P I}$ values) we can not say that the system is out of equilibrium, as the PtBS component is above its effective glass transition when measuring its segmental relaxation by DS for example.

Analogous calculations of $\phi_{\text {self }}^{P t B S}$ for the PtBS component, with the $T_{g, D S C}^{P t B S}$ and $T_{g, D S}^{P t B S}$ data available give values close to zero for its self concentration, $\phi_{\text {self }}^{P t B S} \approx 0$ as previously reported $^{12}$.

The chain length of the two PtBS used is similar to the Kuhn step length for this polymers 
(the Kuhn molecular weight of PtBS, $M_{K} \simeq 1500^{19}$ ). On the one hand, this fact would in principle put into question the strict applicability of the LML model. On the other hand, the result that the effective concentration and the macroscopic concentration are similar can be well rationalized under the LML formalism, where the concentration evaluated in such "large Kuhn volume" is not expected to be very different from the macroscopic one. If the characteristic length relevant for the dynamics of mixtures is redefined, the LML formalism can be successfully applied to describe and rationalize the phenomenology of low molecular mixtures for example ${ }^{20-22}$, for which the model, as originally formulated, would not be strictly applicable. As a consequence, we believe that the LML model may catch the essence of a more general characteristic length concept, which would be related to the Kuhn segment in the case of polymers, but with physical meaning also in other miscible systems.

\section{Nonequlibrium in dilute PI blends}

When the homopolymers forming a blend have very large $T_{g}$ difference, the dynamic asymmetry, i.e. the difference between the characteristic times for each of the components, dramatically increases as temperature decreases till eventually the higher $T_{g}$ component freezes and the system falls out of equilibrium. For the low- $T_{g}$ (or 'fast') component dynamics a crossover behavior is expected corresponding to the transition from a high- $\mathrm{T}$ equilibrium regime to a low-T non-equilibrium regime, where the 'fast' component would move within some kind of frozen network imposed by the 'slow' component. The modified dynamics of the 'fast' component in such a frozen network would consist on more localized motions with low degree of cooperativity for which the literature points towards faster characterisitic times than those expected for the equilibrium state, and an Arrhenius like temperature dependence (see, e.g. reference ${ }^{23}$ for a recent compilation). For a given blend composition, 
these effects on the low- $T_{g}$ component should be more evident the higher the concentration of the high- $T_{g}$ component and the lower the temperature.

A crossover from the equilibrium-like towards confined-like dynamics for the fast component of strongly asymmetric polymer blends was first observed by DS in the system polystyrene(PS)/polyvinylmethylether (PVME) $)^{24,25}$. In that case, the fast component was PVME, which due to its high dielectric strength in comparison with PS, is the responsible of the dielectric response of the PS/PVME blends. It has been reported ${ }^{1}$ that the broad crossover (deviation from the high-T dependence) of the T-dependence of the of the PVME relaxation time takes place in the broad range of the glass-transition of the blend. An Arrhenius regime sets up at the range of the effective glass-transition of PVME in the blend ${ }^{13}$ determined by thermally stimulated depolarization (TSDC) technique (which can be considered the dielectric equivalent to the DSC), and extends well below in temperature ${ }^{1}$. In the case of the PS/PVME blend, the dynamic asymmetry of the system, measured as the difference between the $T_{g}$ s of the two pure components is of the order of $120 \mathrm{~K}$. In our case, the difference between PI's and PtBS's $T_{g}$ s is 126 and $169 \mathrm{~K}$ for PtBS1300 and PtBS2300 respectively. It is expected then that PI's segmental dynamics will be somehow modified by the freezing of the PtBS 'matrix' in those blends with very low PI concentration. In the present case, however, PI/PtBS data do not clearly indicate confined-like dynamics for the fast component PI. Nevertheless the analysis of the data by both DSC and DS techniques for these blends, brings to light some results at odds suggesting that the dynamic behavior of PI in these samples does not correspond with that in equilibrium.

The bigger separation between the $\alpha$ and normal mode relaxation in blends with PI76500 makes these samples very helpful for the characterization of PI's $\alpha$-relaxation regardless the normal mode. As it can be seen in Figure 7 and in contrast to pure PI and PI $>35 \%$ 


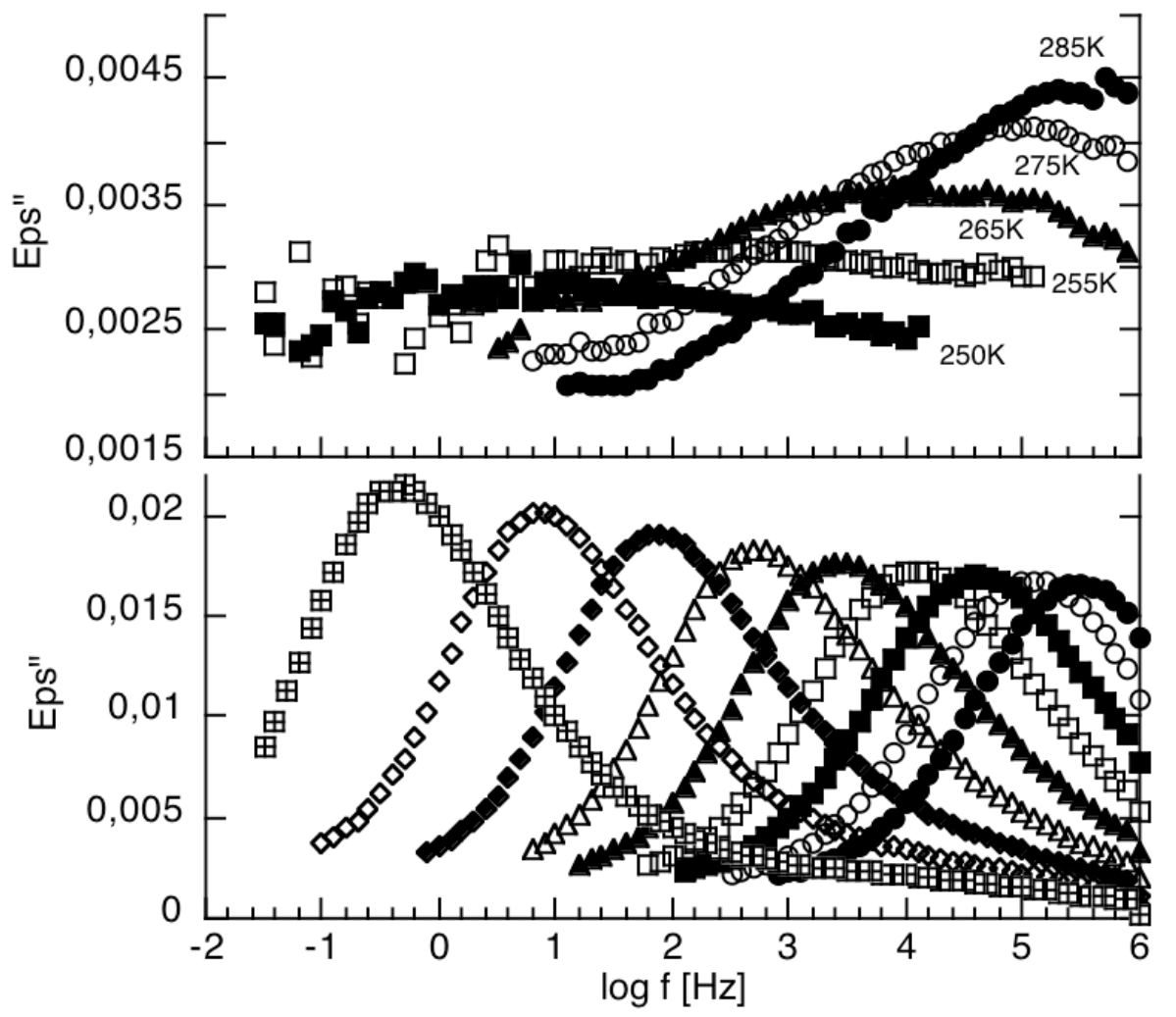

FIG. 7: Upper panel: $\alpha$-relaxation of PI76500 in the blend 20\%PI76500-PtBS2300. Lower panel: $\alpha$-relaxation of pure PI76500 between 215 and $255 \mathrm{~K}$ every $5 \mathrm{~K}$ measured by DS.

blends, the $\alpha$-relaxation of PI in 20\% PI blends shows a marked decrease of its intensity and increase of width as temperature decreases. The observation of very broad relaxation functions is a typical characteristic of confined systems. A decrease of the dielectric strength would be indicative of a decrease in either the number of reorienting dipoles (freezing), or the amplitude of their reorientation (localization of the motion). The low intensity of the $\alpha$-process and the background signal due to the contribution of PtBS, however, prevents from a reliable determination of the relaxation strength, as different evaluations for the 
background produce opposing results.

The comparison of the timescales of the PI $\alpha$-relaxation obtained from the isothermal, $\epsilon^{\prime \prime}(\omega, T=$ const. $)$, and isochronal, $\epsilon^{\prime \prime}(\omega=$ const., $T)$, representations of the dielectric permitivity, also stands out for the lower PI content blends. The characteristic times obtained from the isothermal representation are more physically meaningful. The maxima of $\epsilon$ " $(\omega)$ when plotted as a function of frequency at a constant temperature represents the most probable frequency for the moving units. The isochronal representation, however, gives for a given frequency the temperature at which the intensity of the measured signal is maximum. For PI concentrations $>35 \%$ the characteristic times determined from both representations coincide (see Figure 8). For the blends with $20 \%$ PI, however, the characteristic times obtained from the isothermal representation (which are the well defined ones) are faster than the time-scale deduced from an isochronal representation. This fact is certainly related with the marked variation of the intensity of the $\alpha$-relaxation with temperature for the lower PI content blends. The differences between both representations are negligible for medium and high concentrations and become more and more pronounced as temperature and PI content decrease. This result, i.e. the faster relaxation times obtained in the isothermal representation, could be interpreted and explained as the result of the presence of a faster fraction of "confined" dipoles. This fraction would decrease and its associated characteristic time and dielectric strength become closer to that of the less confined dipoles as temperature increased, making the isochronal relaxation times slower than those observed isothermally.

The extraordinary change of intensity for the segmental relaxation of PI as temperature decreases for the $20 \%$ compositions, may also be the origin of the apparent disagreement between the component dynamics as observed by DS and DSC for low PI content samples. We have seen that DSC and DS results for PI perfectly match for all the samples and composi- 


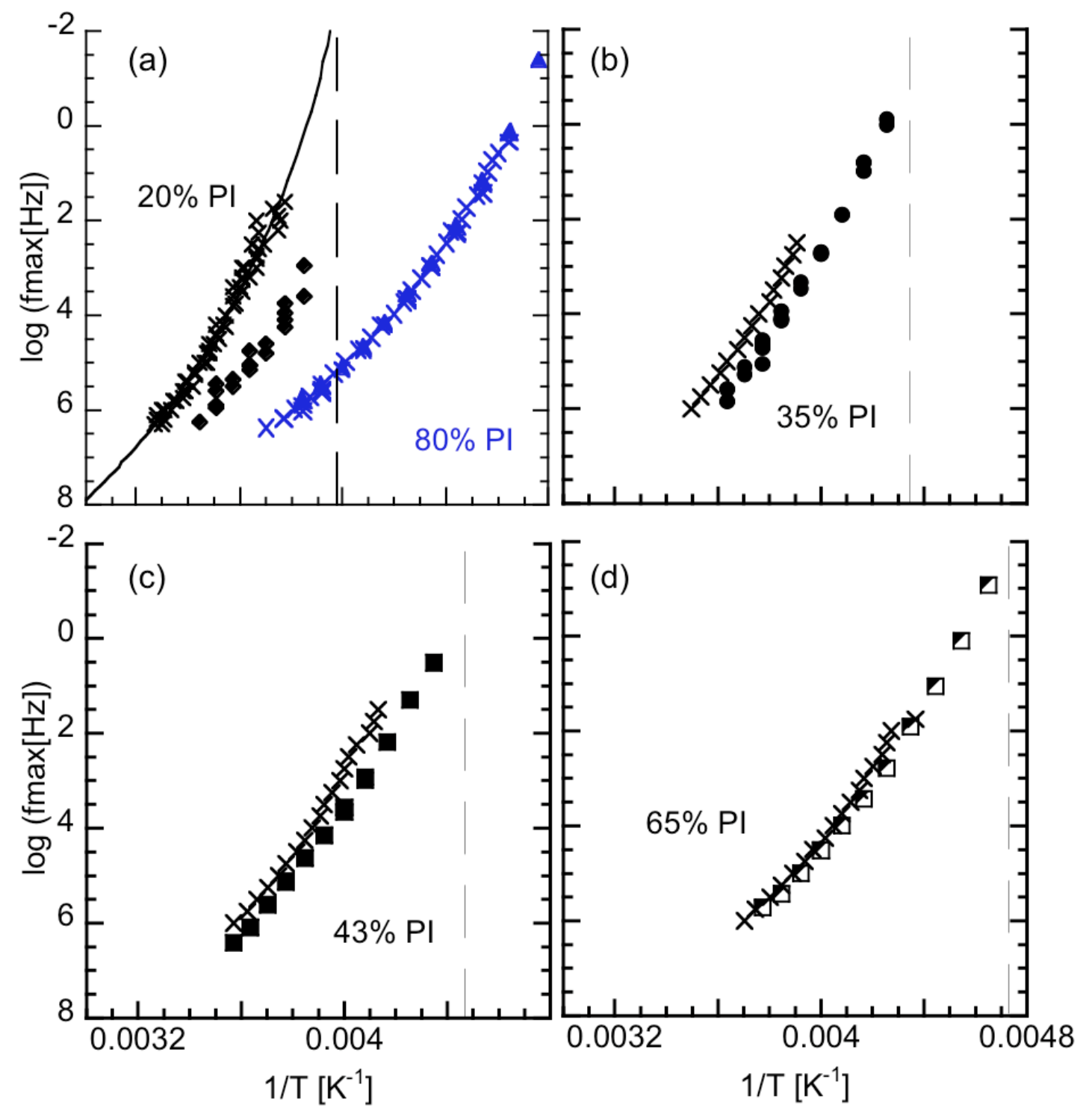

FIG. 8: Characteristic frequencies for the $\alpha$-relaxation of PI in its blends with PtBS2300 obtained from the position of the maxima of $\epsilon$ " in the isothermal (full symbols) and isochronal (crosses) representations for $20 \%$ and $80 \%$-panel (a)-, 35\% -panel (b)-, 43\% -panel (c)-, and 65\% -panel (d)-. Solid line represents the free VFT fitting of the isochronal data. Dashed lines represent the effective glass transition temperatures for PI component determined by DS. 
tions studied except for those with the lowest PI content. In these cases, the dynamics of PI segmental relaxation as seen by DS is faster than that inferred from the calorimetric analysis. Open arrows in Figures 1 and 2 indicate the temperature where $T_{g}^{P I}$ should be in order to describe the experimental dielectric characteristic times obtained from the isothermal representation. Interestingly, the dielectric characteristic times obtained from the isochronal representation predict a component glass transition temperature $T_{g}^{P I}$ that matches that deduced from DSC. Solid line in Figure 8a) represents a free fit of the isochronal points to a VFT law while dashed line represents the component glass transition observed by DSC. It seems, therefore, that the apparent disagreement between the component dynamics as observed by DS and DSC, is no more than the non-equivalence of the temperature and frequency representations when the intensity of the physical processes producing the signal significantly change with temperature ${ }^{26}$. As we have already said, the isochronal representation gives, for a given frequency, the temperature at which the intensity of the measured signal is maximum. At a given frequency, that typically used to define $T_{g}$ for example, $\sim 10^{-2} \mathrm{~Hz}$, both DSC and DS signals reach a maximum value at the same temperature for all the concentration investigated. Even if the physical quantity underlaying DS and DSC techniques is different, it is perfectly consistent that the changes produced by temperature on both signals have the same direction, in fact, a decrease in the dielectric strength due to the localization of the dipolar motion would also produce a decrease of the calorimetric signal, as more local motions produce less changes of entropy or heat capacity.

We note that all the peculiarities observed for low PI content samples are more evident the lower the temperature and PI content, and the higher the difference between homopolymers $T_{g} \mathrm{~s}$, following the expected tendency in case of confinement effects. It is noteworthy that a well defined Arrhenius regime of the PI relaxation time is not evident in figure 8. This 
absence however could be explained taking into account the values of the effective $T_{g} \mathrm{~s}$ of PI in those blends. These values have been marked by dashed lines in figure 8 . They are for the two compositions just in the low-T limit of reliable values of $\tau(T)$. As it has been commented above, the Arrhenius-like regime observed in the case of PVME in PVME/PS blends develops at about the effective $T_{g}$ of PVME in the blend and below. This should also be the expected behaviour in the case of PI in PI/PtBS blends. The absence of $\tau(T)$ data in that low-temperature range would explain why we do not observe the Arrhenius regime observed in other blends. On the other hand, it is evident that representing the broad peaks of figures 1 and 2 by only single values of effective $T_{g} \mathrm{~s}$ is, obviously, an oversimplification. These broad peaks likely represent distribution of $T_{g}$ s associated to almost static composition fluctuations in the system. Figures 1 and 2 also show that the peaks corresponding to PI and PtBS overlap, thereby indicating some overlapping of the $T_{g}$-processes in the crossover region as well. Finally, in the framework of the LML model the high values obtained for $\phi_{\text {self }}^{P I}$ in PI/PtBS would be an indication of a relatively low interdependence of the PI segments with the surrounding host chains of PI/PtBS. This low interdependence implies that very low PI concentration would be needed for the confinement effects to set clear.

However, despite of these large values, our results clearly indicate that in the blends with high concentration of PtBS the PI's segmental dynamics does not follow the trend of those blends in thermodinamical equilibrium, and that the freezing of PtBS chains produces changes in the PI segmental dynamics in the direction of confinement effects.

\section{CONCLUSIONS}

We have observed distinct glass transitions for each component of miscible PI/PtBS blends by calorimetric methods in samples with smaller glass transition temperature dif- 
ference between pure homopolymers than those previously reported for the same system. The comparison of the component glass transition temperatures observed in calorimetry and those obtained from the extrapolation of the PI segmental dynamics in the blend at long times by means of DS is very good, evidencing that the observed double calorimetric signal is really the signature of two distinct glass transitions in the blend. Additionally, the procedure proposed for the calculation of component segmental relaxation times from the calorimetric component glass transitions works pretty well, supporting the suitability of this practice in order to estimate the dynamics of a certain component by means of calorimetric measurements in the cases or systems where dynamic techniques lack selectivity. Finally, the PI segmental dynamics in diluted blends does not follow the trend observed in blends at thermodinamical equilibrium (higher PI concentrations), which could be a signature of confinement effects. However, the low intensity of the signal and the limited experimental window at the low-frequency/low-temperature range where the Arrhenius-like regime should set, prevents from a clearly concluding the presence or absence of this effect.

\section{Acknowledgments}

We acknowledge the Centro de Fisica de Materiales CSIC-UPV/EHU, Donostia International Physics Center, Material Physics Center (MPC), The Basque Government, The Spanish Ministerio de Educacion y Ciencia, The European Commission NoE SoftComp,and the support of the following research projects, grants and contracts: IT-436-07 (GV) , MAT2007-63681, Grant No. CSD2006-53, Contract NMP3-CT-2004-502235,

\footnotetext{
1 Colmenero, J; Arbe, A. Soft Matter, 2007, 3, 1-13.
} 
2 Zawada, J. A.; Ylitalo, C. M.; Fuller, G. G.; Colby, R. H.; Long, T. E. Macromolecules, 1992, $25,2896-2902$.

3 Chung, G.-C.; Kornfield, J. A.; Smith, D. Macromolecules, 1994, 27, 964-973.

4 Chung, G.-C.; Kornfield, J. A.; Smith, D. Macromolecules, 1994, 27, 5729-5741.

5 Alegría, A.; Colmenero, J.; Ngai, K. L.; Roland, C. M. Macromolecules, 1994, 27, 4486-4492.

6 Zawada, J. A.; Fuller, G. G.; Colby, R. H.; Fetters, L. J.; Roovers, J. Macromolecules, 1994, 27, 6851-6860.

7 Arbe, A.; Alegría, A.; Colmenero, J.; Hoffmann, S.; Willner, L.; Richter, D. Macromolecules, 1999, $32,7572-7581$.

8 Sakaguchi, T.; Taniguchi, N.; Urakawa, O.; Adachi, K. Macromolecules, 2005,38, 422-428.

9 Miwa, Y.; Usami, K.; Yamamoto, K.; Sakaguchi, M.; Sakai, M.; Shimada, S. Macromolecules, 2005, 38, 2355-2361.

10 Lodge, T. P.; Wood, E. R.; Haley, J. C. J. Polym. Sci., Part B: Polym. Phys., 2006, 44, $756-763$

11 Gaikwad, A. N.; Wood, E. R.; Ngai, T.; Lodge, T. P. Macromolecules, 2008, 41, 2502-2508.

12 Zhao, J.; Ediger, M. D.; Sun, Y.; Yu, L. Macromolecules, 2009, 42, 6777-6783.

13 Leroy, E.; Alegría, A.; Colmenero, J. Macromolecules, 2002, 35, 5587-5590.

14 Yurekli, K.; Krishnamoorti. R. J. Polym. Sci., Part B: Polym. Phys., 2004, 42, 3204-3217.

15 Watanabe, H.; Matsumiya, Y.; Takada, J.; Sasaki, H.; Matsushima, Y.; Kuriyama, A.; Inoue, T.; Anh, K. H.; Yu, W.; Krishnamoorti, R. Macromolecules, 2007, 40, 5389-5399.

16 Lodge, P.T.; McLeish, T. C. B. Macromolecules, 2000, 33, 5278-5284.

17 He, Y.; Lutz, T. R.; Ediger, M. D.; Pitsikalis, M.; Hadjichristidis, N.; von Meerwall, E. D. Macromolecules, 2005, 38, 6216-6226. 
18 Lutz, T. R.; He, Y.; Ediger, M. D.; Pitsikalis, M.; Hadjichristidis, N. Macromolecules ,2004, 37,6440-6448.

19 Chen, Q.; Matsumiya, Y.; Masubuchi, Y.; Watanabe, H.; Inoue, T. Macromolecules, 2008, 41, 8694-8711.

20 Cangialosi, D.; Alegría, A; Colmenero, J. J. of Chem. Phys., 2007, 126, 204904-204913.

21 Cangialosi, D.; Alegría, A; Colmenero, J. J. of Chem. Phys., 2008, 128, 224508-224518.

22 Cangialosi, D.; Alegría, A; Colmenero, J. Phys. Rev. E, 2009, 80, 041505-041513.

23 ed: Koza, M.; Frick, B.; Zorn, R. Proceeding of the 3rd International Workshop on Dynamics in Confinement. Eur. Phys. J. - Special topics., 2007, 141.

24 Lorthioir, C.; Alegría, A.; Colmenero, J. Phys. Rev. E, 2003, 68, 031805-031814.

25 Urakawa, O.; Sugihara, T.; Adachi, K. Polym. Appl. (Jpn.), 2002, 51, 10.

26 Puértolas, J. A.; Castro, M.; Tellera, I.; Alegría, A. J. Polym. Sci., Part B: Polym. Phys., 1999, $37,1337-1349$. 
TABLE I: Component glass transition for PI2700 and PtBS1300 in their blends from DSC results

\begin{tabular}{ccc} 
PI[\%] & $T_{g}^{P I 2700}(K) T_{g}^{P t B S 1300}(K)$ \\
\hline 100 & 204 & - \\
80 & 211 & - \\
50 & 219 & 240 \\
35 & 228 & 257 \\
20 & 254 & 288 \\
0 & - & 330 \\
\hline
\end{tabular}

TABLE II: Component glass transition for PI2700 and PtBS2300 in their blends from DSC results.

\begin{tabular}{ccc}
\hline $\mathrm{PI}[\%]$ & $T_{g}^{P I 2700}(K) T_{g}^{P t B S 2300}(K)$ \\
\hline 100 & 204 & - \\
80 & 208 & - \\
65 & 212 & \\
43 & 223 & 260 \\
35 & 230 & 276 \\
20 & 251 & 307 \\
0 & - & 373 \\
\hline
\end{tabular}




\section{FIGURE CAPTIONS}

Figure 1: Lower panel: Derivative of the Heat Flow with respect to T for PI2700 and PtBS1300 homopolimers and their blends at different compositions (color on line). The intensity of the curves was multiplied by $0.5,2.5,4$, and 5 , for 100, 50, 35, and $20 \%$ samples respectively. In addition, curves have been shifted in the $y$ axis for clearness. Continuous black lines represent fits to two gaussian functions and dashed lines individual components of the fitting. Upper panel: Glass transition temperature of PI component in the blend as a function of PI content (crosses); horizontal bars represent full width at half maximum of the gaussian fits; dashed line represents the expected global glass transition temperature for the blend according to a simple mixing rule.

Figure 2: Lower panel: Derivative of the Heat Flow with respect to T for PI2700 and PtBS2300 homopolimers and their blends at different compositions (color on line). The intensity of the curves was multiplied by $0.5,0.5,2.5,4$, and 5 , for 100, 80, 43, 35, and 20 $\%$ samples respectively. In addition, curves have been shifted in the y axis for clearness. Continuous black lines represent fits to two gaussian functions and dashed lines individual components of the fitting. Upper panel: Glass transition temperature of PI component in the blend as a function of PI content (crosses); horizontal bars represent full width at half maximum of the gaussian fits; dashed line represents the expected global glass transition temperature for the blend according to a simple mixing rule.

Figure 3: Imaginary part of the dielectric permitivity at 260K for PI2700-PtBS1300 and PI2700-PtBS2300 blends -panels (a) and (b) respectively- at different compositions.

Figure 4: Experimental $\log f_{\max }$ of the PI dielectric $\alpha$-relaxation for pure PI2700 and its blends with PtBS1300 and PtBS2300 - panels (a) and (b) respectively- at different weight

percentages. Solid lines represent the prediction from the $T_{g, D S C}^{P I}$ obtained by DSC. Crosses 
represent the characteristic times obtained from isochronal representation of the data for $20 \%$ PI blends.

Figure 5: Upper panel: Imaginary part of the dielectric permitivity of 20\%PI76500PtBS1300 (filled symbols) and 20\%PI2700-PtBS1300 (empty symbols) blends at 350K. Lower panel: Experimental $\log f_{\max }$ of the PtBS segmental dielectric relaxation for pure PtBS1300 (squares) and its blend with 20\%PI76500 (circles: maxima from isothermal representation, crosses: position of the maxima from an isochronal representation). Solid lines represent the prediction from the $T_{g, D S C}^{P t B S}$ obtained by DSC.

Figure 6: Single component glass transitions $T_{g, D S C}^{P I}, T_{g, D S}^{P I}$ and self concentration $\phi_{\text {self }}$ of PI2700 in its blends with PtBS1300 and PtBS2300 - upper and lower panels respectivelyobtained from both DSC (red: circles $T_{g}$, squares $\phi_{\text {self }}$ ) and DS (blue: crosses $T_{g}$, plusses $\left.\phi_{\text {self }}\right)$ measurements.

Figure 7: Upper panel: $\alpha$-relaxation of PI76500 in the blend 20\%PI76500-PtBS2300. Lower panel: $\alpha$-relaxation of pure PI76500 between 215 and 255K every 5K measured by DS.

Figure 8: Characteristic frequencies for the $\alpha$-relaxation of PI in its blends with PtBS2300 obtained from the position of the maxima of $\epsilon$ " in the isothermal (full symbols) and isochronal (crosses) representations for 20\% and 80\% -panel (a)-, 35\% -panel (b)-, $43 \%$-panel (c)-, and $65 \%$-panel (d)-. Solid line represents the free VFT fitting of the isochronal data. Dashed lines represent the effective glass transition temperatures for PI component determined by DS. 


\section{FOR TABLE OF CONTENTS USE ONLY}

"Comparison of calorimetric and dielectric single component glass transitions in PtBS-PI

blends"

Silvia Arrese-Igor, Ángel Alegría, and Juan Colmenero

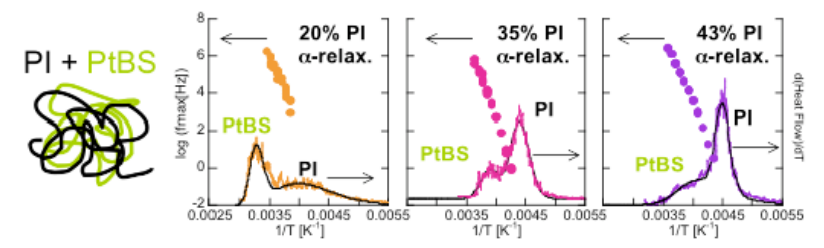

